\title{
Evaluation of the Role of Apoptosis in Vitiligo: Immunohistochemical Expression of P53, Bcl-2 and MART-1 Antigens
}

\author{
Ahmed M. Abdel-Aal*; Mohammad A. Kasem**and Ahmed H. Abdel-Rahman*** \\ From the Department of Dermatology, Venereology \& Andrology, Al-Azhar University in \\ Cairo*; Departments of Histology** and Pathology***, Al-Azhar University, Assuit
}

\begin{abstract}
It is clear that melanocytes are absent from the vitiliginous skin. However, the mechanism of melanocyte disappearance has never been clearly understood. It is not clear whether melanocyte loss in vitiligo results from apoptosis or other causes of cell death. A very few data were published about the role of apoptosis in vitiligo.

To investigate the role of apoptosis in vitiligo, ten biopsies from patients and ten normal control biopsies were taken from the skin of the thigh by the scalped biopsy technique. Each biopsy included adjacent areas of the lesional, peri-lesional and non-lesional skin. All subjects were males selected at Al-Housein University Hospital and ranged between 25 to 43 years in age.

The expression of MART-1 protein as a melanocyte marker, Bcl-2 as an antiapoptotic molecule and P53 as a proapoptotic molecule were evaluated by the immunoperoxidase technique and computerized image analysis. The study revealed complete absence of MART-1 positive melanocytes in the lesional skin, gradual appearance in the peri-lesional areas and showed no significant difference between the non-lesional areas and the normal skin $(\mathrm{P}=0.43)$. The melanocytic Bcl-2 expression was higher in normal skin than the non-lesional area, lower in the peri-lesional areas and lowest in the lesional areas $(\mathrm{p}<0.05)$. Conversely, more expression of melanocytic P53 was seen in the lesional than the peri-lesional areas, lower in the nonlesional skin and lowest in the normal skin $(\mathrm{p}<0.05)$. Accordingly, the study revealed more susceptibility to apoptosis in the vitiliginous skin than in normal skin. We hope that these data can help in understanding the pathogenesis and the progression of this disease as well as providing better lines of its treatment.
\end{abstract}

Key words: Apoptosis, Bcl-2, P53, Immunohistochemistry, Melanocytes, Vitiligo.

\section{Introduction:}

Vitiligo is an acquired progressive skin disorder characterized by depigmentation caused by selective destruction of melanocytes which are highly specialized cells responsible for skin pigmentation ( $L e$ Poole et al., 1993). Clinically, vitiligo is manifested as white macuoles on the skin that can be localized or generalized. The course of the disease is unpredictable but is often progressive with phases of stabilized depigmentation (Castanet and Ortonne, 1997).

Normally, the epidermis has a stratified, keratinizing epithelium consisting of keratinocytes (85-90\%) and nonkeratinocyte cells usually with a dendritic morphology, representing $10-15 \%$ of the total cell population; these include Langerhans cells, melanocytes and Merkel cells. Melanocytes are cells with a dendritic morphology, of neural crest origin located within the basal cell layer. The ratio of melanocytes to basal keratinocytes along the dermoepidermal junction is site dependent and ranges from approximately 1:4 in the cheek to 1:11 in the thigh (Breathnach, 1971). They label on routinely-processed tissue sections with antibodies to MART-1 protein (Kanitakis, 1998, Palermo et al, 2001 and Carlson et al., 2002). Melanocytes also constitutively express bcl-2 oncoprotein; a $25 \mathrm{kDa}$ cytoplasmic 
protein encoded by an oncogene preventing cells from undergoing apoptosis (Van der Ord et al., 1994 and Kanitakis et al., 1995), the c-kit protein (Norris et al., 1996) and tyrosinase. On the other hand, melanocytes and Langerhans cells are the only intraepidermal cells expressing vimentin (Kanitakis, 1998).

Numerous hypotheses about the etiology of vitiligo had been found however, none of them was proven. One way to understand the etiology was to determine the mechanism by which melanocytes were destroyed. Two known mechanisms for this cell loss were found, necrosis and apoptosis (Huang et al, 2002). The histology of vitiligo strongly suggests apoptosis rather than necrosis as the mechanism for melanocyte destruction (Biossy et al, 1991 and Abdel-Naser et al, 1994).

Apoptosis or programmed cell death is modulated by a set of genes within the cells and considered as an important mechanism for control the number and location of cells. The removal of the cells is performed in a highly controlled, a very precise and a discriminating manner as the by-products from the cell constituents are hidden from the immune system and do not initiate an immune response like in necrosis. In addition, apoptosis is initiated either within the cell or by outside signals (Malmusi and Ackerman, 2000).

Several proteins of the Bcl-2 family including Bcl-2 itself, Bcl-x1 and Bcl-w, act as antiapoptotic protecting the cell from destruction. On the other hand, the P53 proteins function as proapoptotic inducing the cell into apoptosis; these two sets of molecules antagonize and balance each other (Huang et al., 2002).

Certain factors may induce apoptosis in the epidermis either by suppressing expression of $\mathrm{Bcl}-2$ and/or enhance the expression of P53 such as the ultraviolet light especially ultraviolet B (UVB) (Okamoto et al, 1999 and Van Den Wijngaard et al, 2000). It might be postulated that those individuals with genetically low susceptibility to suninduced cancers, are protected via the P53 mechanism. They have a low threshold for induction of apoptosis and are prone to vitiligo but resistant to skin cancers. That description fits, at least, a large population of vitiligo patients (Leffell, 2000 and Mc Kay et al, 2000).

The aim of the work is to study the role of apoptosis in vitiligo through investigating the levels of expression of Bcl-2 and P53 as they maintain the balance against or toward cell death. In this study, we compared the lesional skin to the perilesional, non-lesional and the normal skin as well.

\section{Subjects \& Methods}

The study was performed on two groups:

- Group-1: Included 10 vitiligo patients (generalized vitiligo vulgaris).

- Group-2: Included 10 normal control subjects.

Cases of group-1 were selected at the Department of Dermatology, while cases of group-2 were selected at the Department of Plastic Surgery from patients attending for cosmetic problems, at Al-Housein University Hospital with consent.

Each biopsy was taken by the scalped biopsy technique from the skin of the thigh including lesional, peri-lesional and nonlesional areas. All cases ranged between 25 to 43 years in age. Specimens were formalin-fixed, paraffin-embedded and cut at $6 \mathrm{~m} \mu$ and subjected to the following techniques:

\section{1) Haematoxylin and Eosin (H\&E) staining:}

The staining was done to explore the morphologic appearance of the skin and to ensure the integrity of the specimens before the immunoperoxidase technique.

2) The immunoperoxidase

(IP) technique:

According to the manufacturer instructions of the UltraVision Large Volume Detection System (Lab-Vision CA. USA, Catalog Number TP-060-HL), immunoperoxidase method for detection of MART-1 protein as a specific melanocyte marker, Bcl-2 as an anti-apoptotic protein and P53 as a pro-apoptotic protein were done.

Paraffin sections were downed to water. The endogenous peroxidase activity was blocked by incubating the sections in hydrogen 


\section{Ahmed M Abdel-Aal et al}

peroxide for 10 minutes and washed twice in phosphate buffered saline (PBS). Enzyme digestion was performed by incubating the sections in $0.1 \%$ trypsin solution at $37^{\circ} \mathrm{C}$ for 10 minutes followed by 4 changes in PBS. To block nonspecific background staining, sections were incubated in the "Ultra V Block" for 5 minutes at room temperature followed by rinsing in PBS. The sections were incubated in the primary monoclonal antibody according to table 1 :

\section{Table 1: Types and application of monoclonal antibodies}

\begin{tabular}{cccc}
\hline Antibody & Company & Catalog No & Incubation \\
\hline MART-1 & Zymed Lab. & $18-7238$ & overnight \\
Bcl-2 & NeoMarkers & MS-123-R7 & overnight \\
P53 & Dako & M7001 & overnight \\
\hline
\end{tabular}

The sections were washed 4 times in PBS, then, incubated in the biotinylated Goat Anti-Polyvalent solution for 10 minutes at room temperature followed by 4 changes in PBS. The streptavidin peroxidase was applied for 10 minutes at room temperature, then sections were washed 4 times in PBS. Finally the sections were incubated with the chromogen substrate for 10 minutes, counterstained with haematoxylin and coversliped.

\section{Quantitative Analysis}

All sections were examined after staining by the computerized image analysis. The mean percentage of positive areas to the total epidermal areas was calculated using Image Pro Plus V4.51 (Media Cybernetics Inc. 2002).

The statistical analysis was carried out according to the standard statistical procedures and Student (t) test to estimate the significance of results using Microsoft Excel XP 2002.

\section{Results}

\section{Morphological Examination:}

Haematoxylin and eosin (H\&E) staining revealed absolute loss of melanocytes in the lesional areas confirmed by the immunohistochemistry of MART- 1 . There was more availability of melanocytes in the non-lesional than in perilesional areas. The epidermis of the lesional areas showed also a relative lack of regular arrangement and cell distribution in the epidermis than the non-lesional areas (Fig. $4)$.

\section{Molecular Expression:}

Microscopic examination revealed complete absence of MART-1 positive melanocytes in the lesional skin, and more availability in the non-lesional than in perilesional areas. The later showed gradual loss of positive cells toward the lesional area $(\mathrm{P}<0.05)$. There was no significant difference between the normal control and the non-lesional skin $(\mathrm{P}=0.43)$ (Table 2, Fig. 1\&5).

The melanocytic Bcl-2 expression was higher in normal skin than the nonlesional area, lower in the peri-lesional areas and lowest in the lesional areas $(p<0.05)$. The peri-lesional areas showed gradual loss of positive cells toward the lesional area (Table 3, Fig. 2\& 6).

Conversely, more expression of melanocytic P53 was seen in the lesional than the peri-lesional areas, lower in the non-lesional skin and lowest in the normal skin $(p<0.05)$. The peri-lesional areas showed gradual increase of positive cells toward the lesional area (Table4, Fig. 3\& 7). 
Table 2: The Mean Percent of MART-1 Positive area to the total Epidermal area

\begin{tabular}{|l|l|l|l|l|l|l|}
\hline & 1 & 2 & 3 & 4 & \\
\cline { 2 - 7 } & Cont & N-L & Peri-L & \multicolumn{2}{l|}{ Lesional } & \\
\hline Average & 4.422 & 4.309 & 2.78 & 0 & & \\
\hline SD & 0.27 & 0.42 & 0.19 & 0 & $3-4$ & $2-4$ \\
\hline Gr-Comp & $1-2$ & $2-3$ & $1-3$ & $1-4$ & $<0.00001$ & $<0.00001$ \\
\hline P-Value & 0.43 & 0.009 & 0.011 & $<0.00001$ & $<$ \\
\hline
\end{tabular}

Table 3: The Mean Percent of BCL2 Positive area to the total Epidermal area

\begin{tabular}{|l|l|l|l|l|l|l|}
\hline & 1 & 2 & 3 & 4 & \multicolumn{3}{l|}{} \\
\cline { 2 - 7 } & Cont & N-L & Peri-L & Lesional & & \\
\hline Average & 4.035 & 3.8 & 2.085 & 1.042 & & \\
\hline SD & 0.19 & 0.21 & 0.13 & 0.16 & -4 \\
\hline Gr-Comp & $1-2$ & $2-3$ & $1-3$ & $1-4$ & $3-4$ & 0.003 \\
\hline P-Value & 0.00015 & 0.0006 & 0.007 & 0.00014 & 0.0011 & 0.06 \\
\hline
\end{tabular}

Table 4: The Mean Percent of P53 Positive area to the total Epidermal area

\begin{tabular}{|l|l|l|l|l|l|l|}
\hline & 1 & 2 & 3 & \multicolumn{4}{|l|}{4} \\
\cline { 2 - 7 } & Cont & N-L & Peri-L & Lesional & \\
\hline Average & 0.095 & 0.108 & 0.329 & 0.422 & \\
\hline SD & 0.0002 & 0.001 & 0.001 & 0.0007 & $3-4$ & $2-4$ \\
\hline Gr-Comp & $1-2$ & $2-3$ & $1-3$ & $1-4$ & 0.0006 & 0.00013 \\
\hline P-Value & 0.08 & 0.0011 & 0.0003 & 0.000001 & 0.00 \\
\hline
\end{tabular}

Gr-Comp $=$ groups compared, Con $=$ control, $N-L=$ non-lesional, $P-L=$ peri-lesional, $L=$ lesional, $S D=$ standard deviation

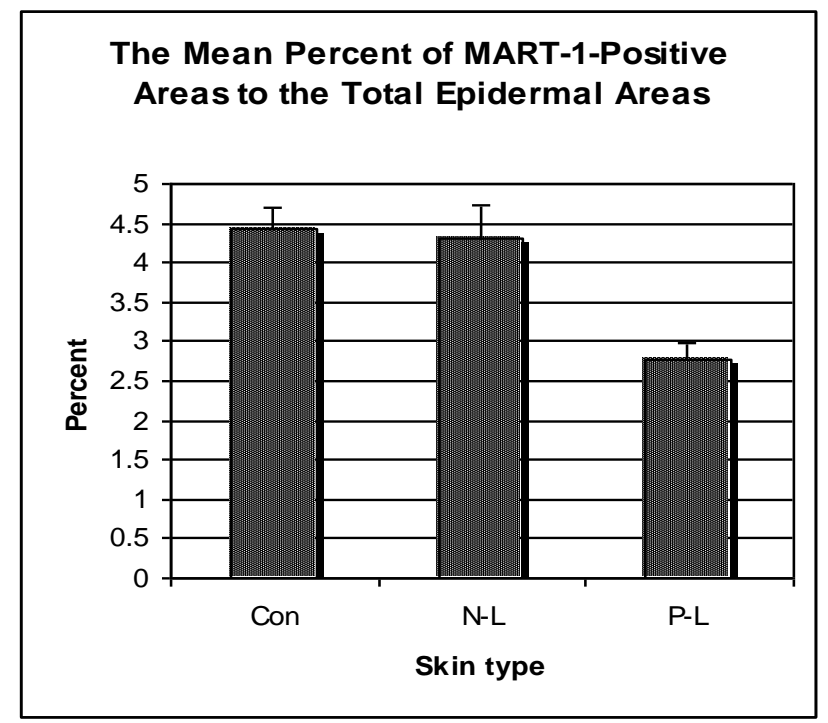

Figure 1

The lesional skin was not included in the graph since it was quite negative to the MART-1 
The Mean Percent of Bcl2-Positive Areas to the Total Epidermal Areas

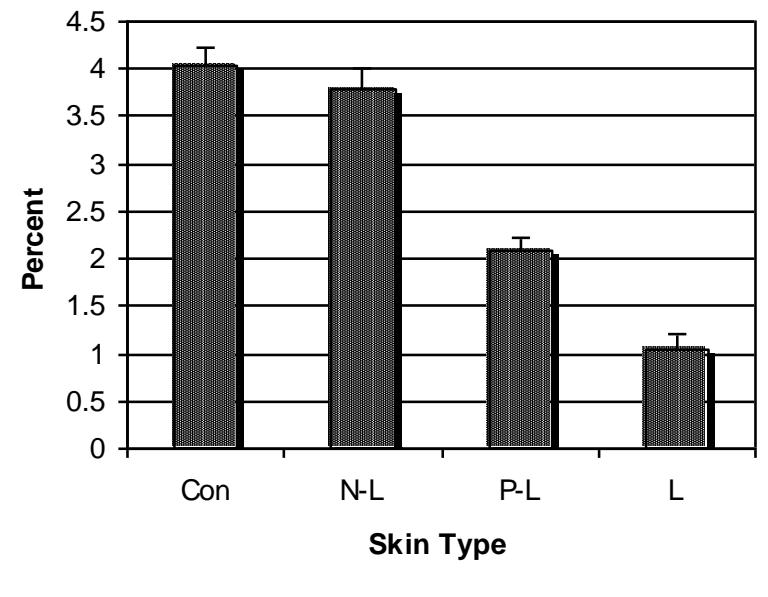

Figure 2

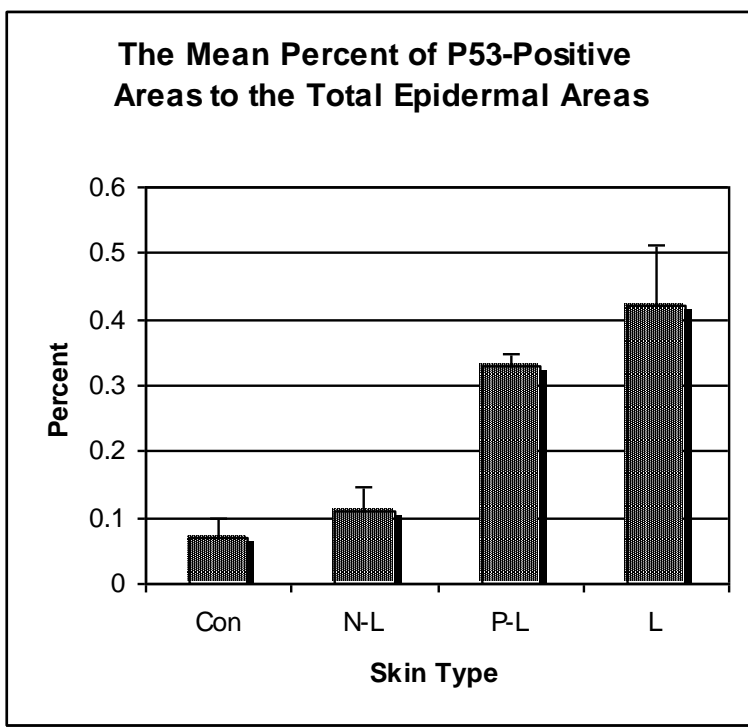

Figure 3

Con $=$ control, $N-L=$ non-lesional, $P-L=$ peri-lesional, $L=$ lesional Error bars represent the standard deviation (SD). 

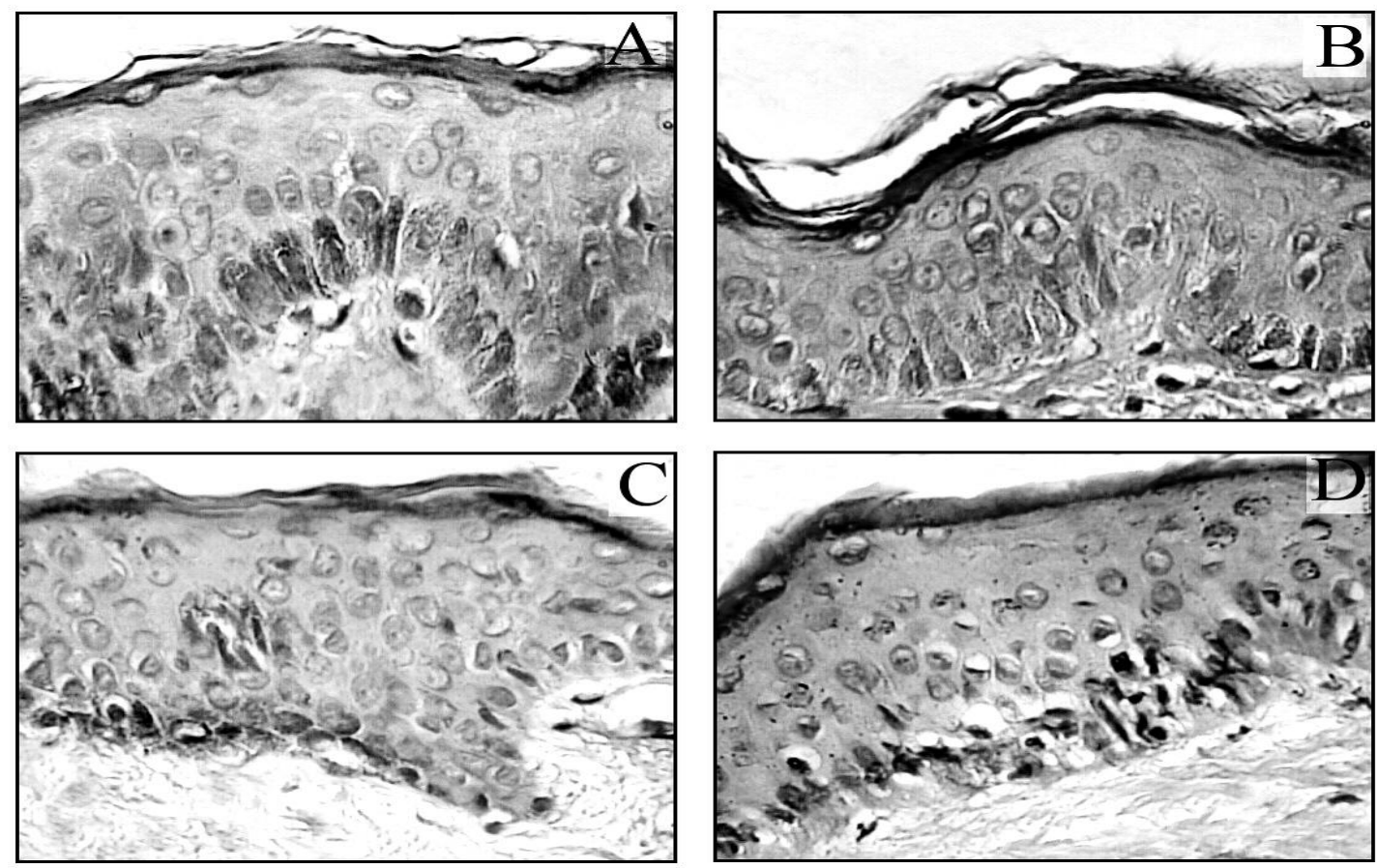

Figure (4)

The Morphological appearance of the skin after $H \& E$ staining. In normal skin (A), melanocytes are present and the epidermal cells are organized. In the non-lesional skin $(B)$, the epidermis is relatively similar to the control. In the peri-lesional area there is gradual loss of melanocytes (C) which are totally lost in the lesional area with disorganization of the epidermal layers $(D)$.

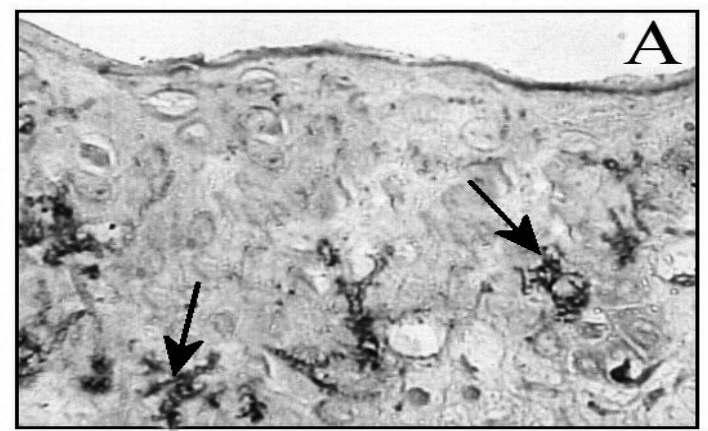

$(H \& E, x 400)$
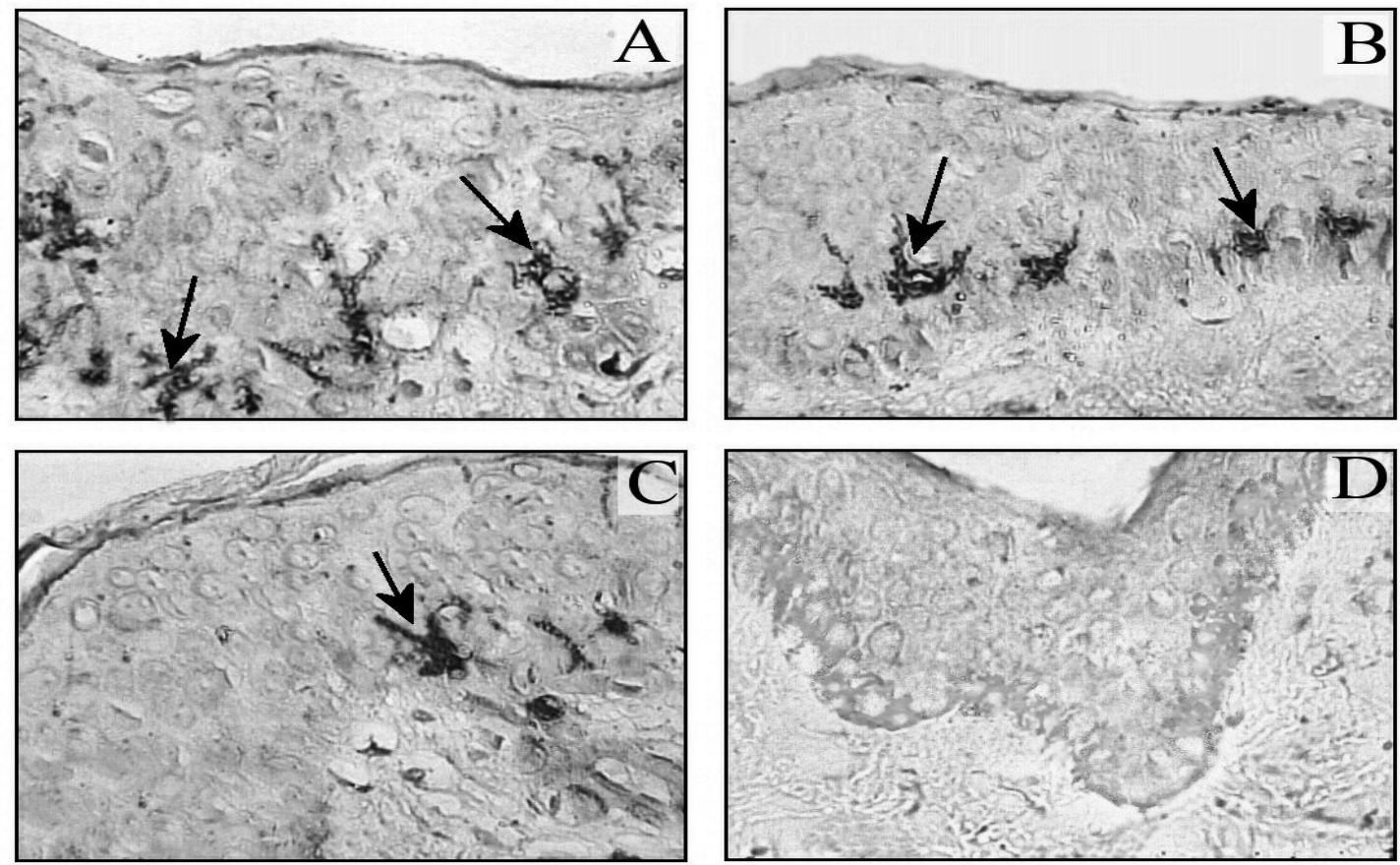

Figure (5)

Immunohistochemical labeling of melanocyte with MART-1 showing normal pattern in the control (A) and similar picture in the non-lesional skin (B), gradual loss in the peri-lesional area $(C)$ and total absence in the lesional skin $(D)$.

(Immunoperoxidase (IP) $x 400$ ). 

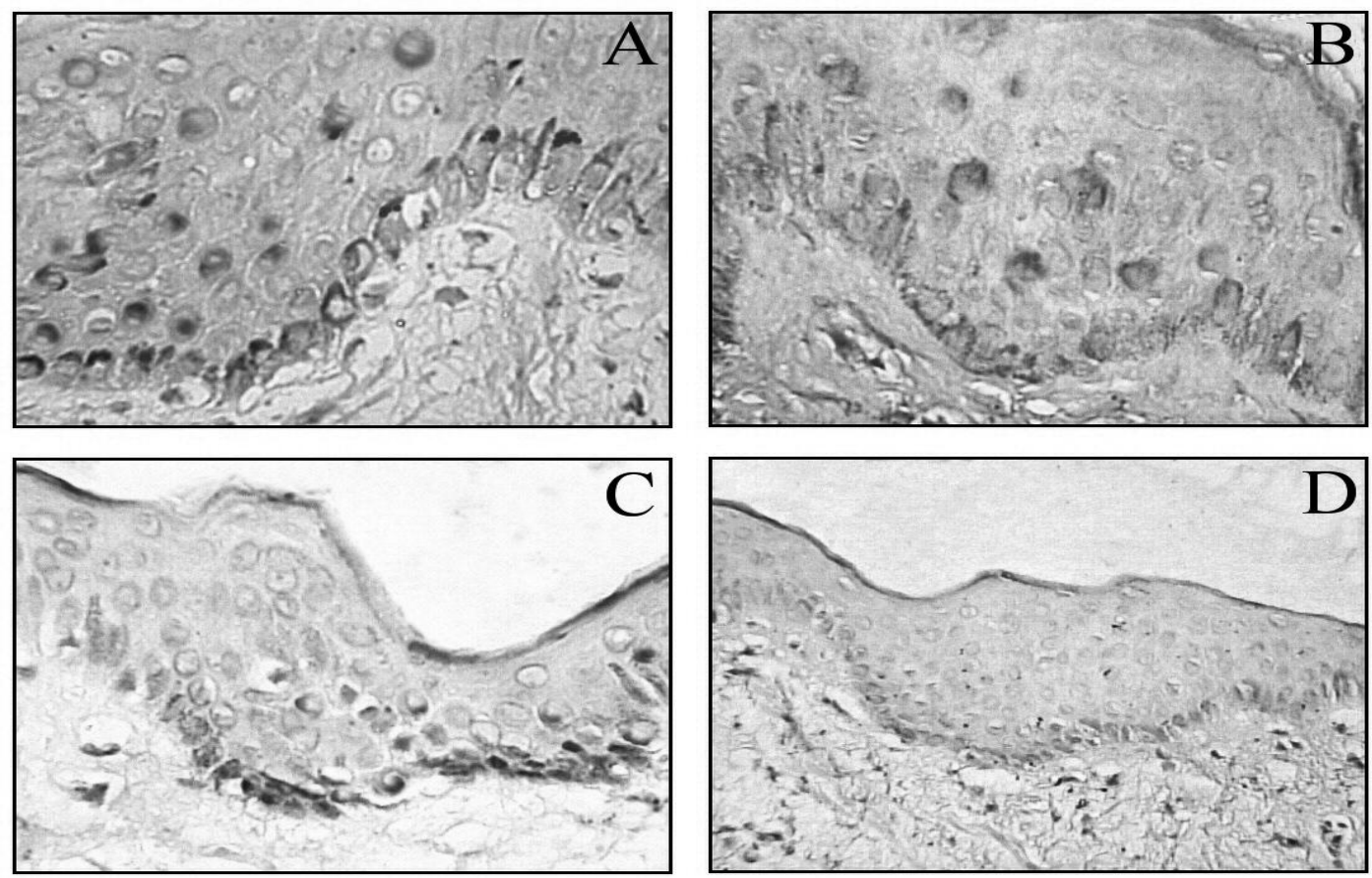

Figure (6)

The expression of Bcl-2 protein showing a relatively higher level of expression in the control (A) and slightly lower level in the non-lesional skin (B), gradual loss in the peri-lesional area (C) and maximal derease in the lesional skin (D) (IP X 400).
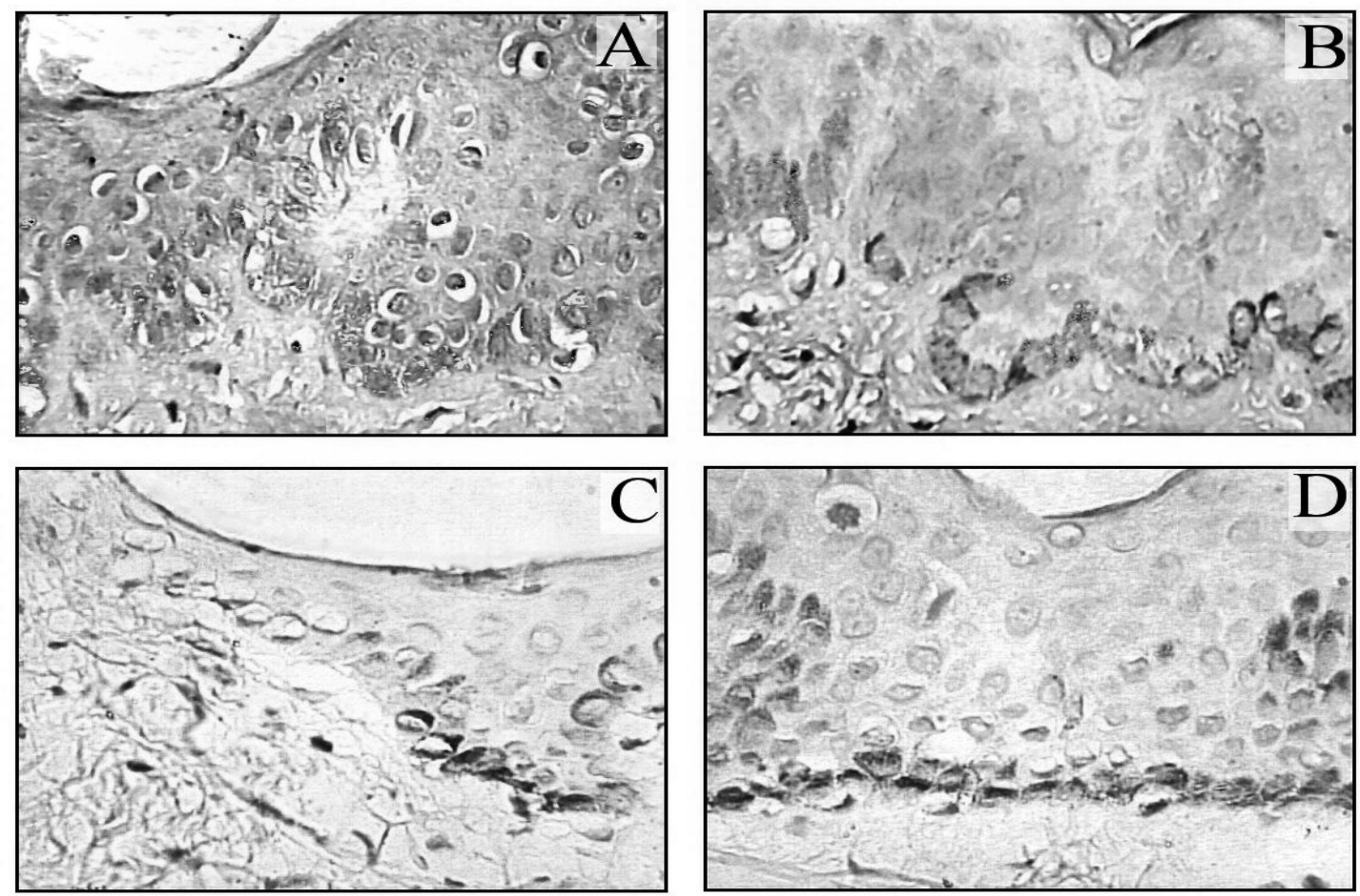

Figure (7)

The expression of P-53 protein showing lowest levels of expression in the control (A) and slightly higher level in the non-lesional skin $(B)$, gradual increase in the peri-lesional area $(C)$ and the highest levels in the lesional skin (D).

(IP X 400). 


\section{Discussion:}

Vitiligo is a skin disease characterized by white spots that usually progress with enlargement of the existing lesions and appearance of new ones. The cause is the selective destruction of melanocytes that are absent in established lesions. In the normal skin, melanocytes are regularly and predictably arrayed in the epidermis along the dermoepidermal junction (Breathnach, 1971).

The melanocyte-specific melanosomal-related protein, Mart-1 (Melan-A) is an antigen recognized by autologous cytotoxic T cells (Kawakami et al, 1994 \& 1997 and Chen et al, 1996). Our study showed complete absence of MART-1 positive melanocytes in the lesional skin coinciding with that reported by Le Poole et al, (1993) and Castanet \& Ortonne 1997; who also stated that the melanocytic loss is thought to be due to their destruction in-situ.

Carlson et al, 2002 suggested that there are many melanocytes (melanogenic and nonmelanogenic) within the epidermis that cannot be detected by standard methods. Some of these Mart-1-labeled melanocytes may represent the so-called precursor melanocytes that were detected by Grichnik et al, 1996. The absence of melanocytes demonstrated in most studies of vitiligo may be partially due to searching for functioning melanocytes with active melanogenesis (Le Poole et al, 1993; Bhawan, 1997; Norris et al, 1996; and Hann et al, 2000). Inactive melanocytes may exist in vitiligo that awaits the necessary signals or environmental change to begin the normal melanogenesis (Carlson et al, 2002).

As an acute inflammatory response is not a general finding in vitiligo (Le Poole et al, 1996), it was hypothesized that melanocytes die in a controlled manner via apoptosis (Cohen, 1993). Because melanocytes in patients with vitiligo may intrinsically be more susceptible to apoptotic stimuli, we compared melanocytes from control individuals to those of vitiligo patients through expression of Bcl-2 and P53. Apoptosis is induced when the anti-apoptotic factors are overcome by lethal stimuli or loose their natural defenses. Newer reports stated that the melanocytic cell death in vitiligo, particularly the generalized type, occured in the absence of neutrophil influx and erythema, suggesting apoptosis (Rudin \& Thompson 1997 and Huang et al, 2002).

Bcl-2 was investigated because of its important opposing role in regulating the susceptibility to apoptosis (Van Den Wijngaard et al, 2000). In accordance with earlier reports (Plettenberg et al, 1995; Saenz-Santamaria et al, 1994), in-situ analysis revealed high melanocytic bcl-2 expression in normal skin. Equally, high bcl-2 expression was observed in the nonlesional skin (Van Den Wijngaard et al, 2000). In this work, we have detected a comparatively higher value for bcl-2 expression in the normal control skin than other investigated regions.

The expression of bcl-2 can be regulated by the tumor suppressor gene P53 (Miyashita et al, 1994) and it is known that P53 is involved in inducing apoptosis. Our study showed that P53 - expression was higher in the lesional and perilesional sites than in the control specimens. Moreover, the mean percent of melanocytic P53 positive areas showed a significant increase in the lesional skin compared to other areas ( $\mathrm{P}<0.05)$, indicating more susceptibility to apoptosis. These findings are identical to those observed by Campbell et al, 1993, who reported an increase in the p53 expression, in the human skin after exposure to ultraviolet (UV) rays It was also shown that UV is capable of modulating melanocyte-expressed apopto-sis regulatory molecules leading to loss of melanocytes (Van Den Wijngaard et al, 2000).

It was reported that infiltrating $\mathrm{T}$-cells and macrophages can be observed adjacent to the remaining peri-lesional melanocytes in generalized vitiligo and it was shown that cytotoxic T-cells, macrophages are both capable of inducing apoptosis of melanacytse in vitro . Therefore, it is feasible that in vivo melanocyte apoptosis in vitiligo is caused by auto-reactive T-cell and/or 
macrophages (Van Den Wijngaard et al, 2000). However, apoptosis might be an alternative way to view the role of immune system and its involvement in melanocyte destruction.

\section{Conclusion}

Our data strongly supported apoptosis rather than necrosis as a mechanism for the elimination of melanocytes in vitiligo. Therefore, the factors that can tilt the balance away from the pro-toward the antiapoptotic control may prevent the spread of the disease. Accordingly, the drugs that can enhance the anti-apoptotic proteins e.g. bcl2 or suppress the expression of proapoptotic ones e.g. P53 may provide a raical solution of this disorder.

\section{References:}

1. Abdel-Naser MB; Kruger-Krasagakes S. and Krasagakes K. (1994): Further evidence for involvement of both cell mediated and humoral immunity in generalized vitiligo. Pigment Cell Res., 7 (1): 1-8.

2. Bhawan ,J. (1997): Mel-5: A novel antibody for differential diagnosis of epidermal pigmented lesions of the skin in paraffin-embedded sections. Melanoma Res; 7:43-8.

3. Biossy RE, Liu YY, Medrano EE and Nordlund J.J. (1991): Structural abberation of the rough endoplasmic reticulum and melanosome compartmentalization in long-term cultures of melanocytes from vitiligo patients. J. Invest. Dematol., 97 (3): 395-404.

4. Breathnach A.S. (1971): An atlas of the ultrastructure of the skin. London: J \& A Churchill: 136.

5. Campbell C, Quinn AG, Angus B, Farr PM and Rees JL. (1993): Wavelength specific patterns of p53 induction in human skin following exposure to UV radiation. Cancer Res.; 53: 2697-9.

6. Carlson JA; Grabowski R; Mu XC; Del Rosario A; Malfetano J and Slominski A (2002): Possible Mechanisms of Hypopigmentation in Lichen Sclerosus. Am. J. Dermatopathol; 24:97-107.

7. Castanet J and Ortonne JP (1997): Pathophysiology of vitiligo. Clin. Dermatol; 15: 845-51.
8. Chen YT; Stockert $\mathbf{E}$ and Jungbluth A (1996): Serological analysis of Melan-A (MART-1), a melanocyte-specific protein homogeneously expressed in human melanomas. Proc Natl Acad Sci USA; 93:5915-9.

9. Cohn JJ (1993): Apoptosis. Immunol. Today; 14: 131-6.

10. Grichnik JM; Ali WN and Burch JA (1996): KIT expression reveals a population of precursor melanocytes in human skin. J Invest Dermatol; 106:967-71.

11. Hann SK; Kim YS and Yoo JH (2000): Clinical and histopathologic characteristics of trichrome vitiligo. J Am Acad Dermatol; 42:589-96.

12. Huang CL; James JN and Raymond B (2002): Vitiligo: A manifestation of apoptosis? Am. J. Clin. Dermatol; 3 (5): 301-8.

13. Kanitakis,.(1998): Immunohistochemistry of normal human skin. Eur J Dermatol. 8 (8) : 539-47.

14. Kanitakis J; Montazeri A; Ghohestani R; Faure M and Claudy A (1995): Bcl-2 oncoprotein expression in benign nevi and malignant melanomas of the skin. Eur $\mathbf{J}$ Dermatol; 5:501-7.

15. Kawakami Y; Battles JK and Kobayashi T (1997): Production of recombinant MART-1 proteins and specific antiMART1 polyclonal and monoclonal antibodies: Use in the characterization of the human melanoma antigen MART-1. J Immunol Methods; 202:13-25.

16. Kawakami Y; Eliyahu S and Sakaguchi K (1994): Identification of the immunodominant peptides of the MART-1 human melanoma antigen recognized by the majority of HLA-A2-restricted tumor infiltrating lymphocytes. J Exp Med; 180:347-52

17. Le Poole IC; Van Den Wijnaard R.M, Westerhof, W; Dutrieux RP and Das PK (1993): Presence or absence of melanocytes in vitiligo lesions: An immunohistochemical Investigation. J. invest. Dermatol.; 100: $816-22$

18. Le Poole IC; Van Den Wijngaard RM; Westerhof W and Das PK (1996): Presence of $\mathrm{T}$ cells and macrophages in inflammatory vitiligo skin parallels melanocyte disappearance. Am J Pathol; 148: 1219-28.

19. Leffell D (2000): The scientific basis of skin cancer J. Am. Acad. Dermatol.; 42 (1pt 2): 18-22. 
20. Malmusi M and Ackerman A (2000): A critical review of apoptosis in historical perspective. Am. J. Dermatopathol.; 22: 291-3.

21. Mc Kay B; Chem F. and Perumalswami CR (2000): The tumor suppressor P53 can both stimulate and inhibit ultraviolet lightinduced apoptosis. Mol. Biol. Cell; 11: 2543-51

22. Miyashita T; Krajewski S. and Krajewska M. (1994): Tumor suppressor p53 is a regulator of bcl-2 and bax gene expression in vitro and in vivo. Oncogene; 9: 1799-805.

23. Njoo MD and Westerhoof W (2001): Vitiligo: Pathogenesis and treatment. Am. J. Clin. Dermatol; 2: 167-81.

24. Norris A; Todd C; Graham A; Quinn A and Thoddy A (1996): The expression of the c-kit receptor by epidermal melanocytes may be reduced in vitiligo. $\mathrm{Br} \mathrm{J}$ Dermatol; 134: 299-306.

25. Okamoto H; Mizuno $K$ and Itoh $T$ (1999): Evaluation of apoptotic cells induced by ultraviolet light $\mathrm{B}$ radiation in epidermal sheets stained by the TUNEL technique. J. Invest. Dermatol; 113: 802-7.

26. Palermo B, Campanelli R, Garbelli $\mathbf{S}$ Mantovani S, Lantelme E, (2001): Specific Cytotoxic T-Lymphocyte Responses Against Melan-A/MART1,
Tyrosinase And Gp100 In Vitiligo By The Use Of Major Histocompatibility Complex/Peptide Tetramers: The Role Of Cellular Immunity In The Etiopathogenesis Of Vitiligo. J Invest Dermatol 117 (2): 326332.

27. Plettenberg A; Ballaun $\mathbf{C}$ and Pammer $\mathbf{J}$ (1995): Human melanocytes and melanoma cells constitutively express the Bcl-2 protooncogene in-situ and in cell culture. Am. J. Pathol.; 146: 651-9.

28. Rudin CM and Thompson CB (1997): Apoptosis and disease Regulation and clinical relevance of programmed cell death. Annu. Rev. Med.; 48: 267-81.

29. Saenz-Santamaria MC; Reed JA; Mc Nult NS and Shea CR (1994): Immunohistochemical expression of Bcl-2 in melanomas and intradermal nevi; $\mathrm{J}$. Cutan, Pathol, 21: 393-7.

30. Van Den Wijngaard RM; Aten J and Scheepmaker A (2000): Expression and modulation of apoptosis regulatory molecules in human melanocytes: Significance in vitiligo. Br. J. Dermat.; 143: 573-81.

31. Van der Ord J; Vandenghiste N; De Ley $M$ and de Wolf-Peters C (1994): Bcl-2 expression in human melanocytes and melanocytic tumors. Am J Pathol; 145: 294-300. 
تقييم دور التساقط الخلوى فى مرض البهاق : دراسة بالكيمياء النسيجية المناعية

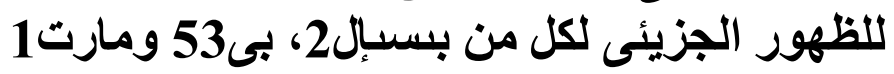

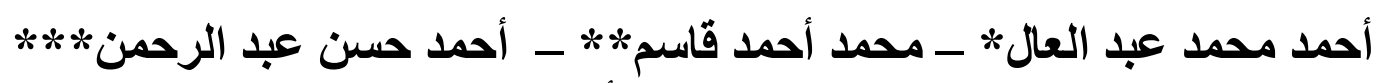

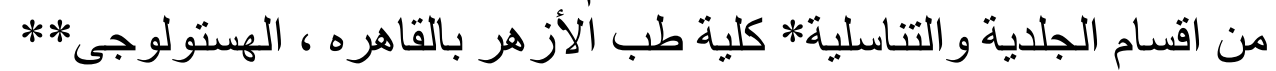
و الباثولوجى *** كلية طب الأز هر - فرع اسليوط

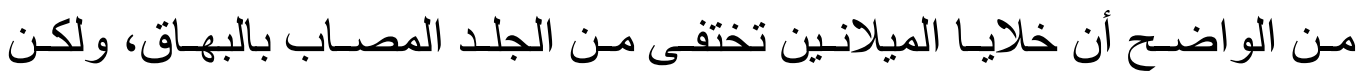

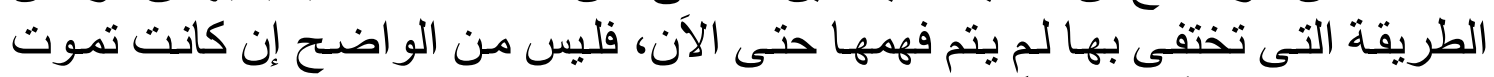

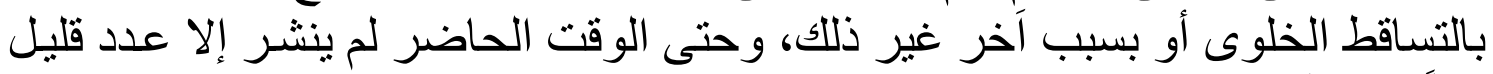

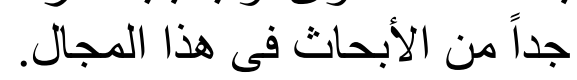

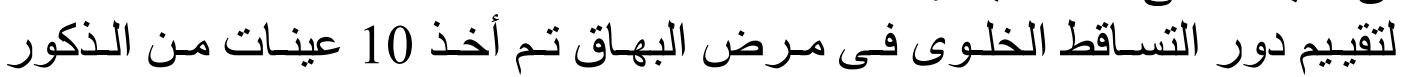

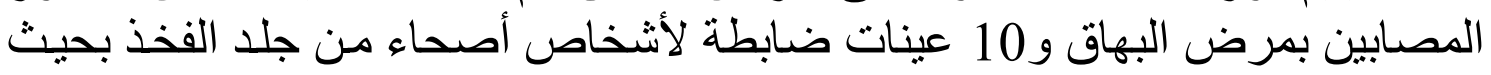

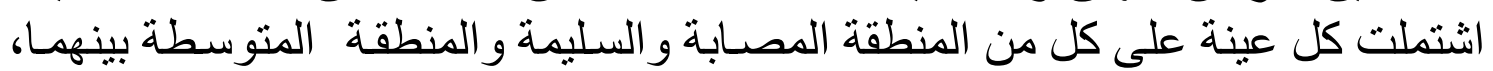

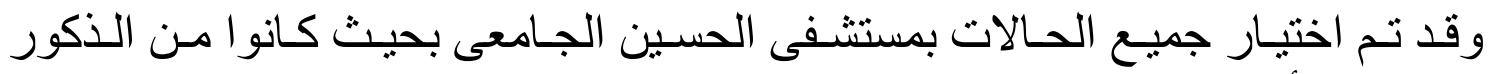

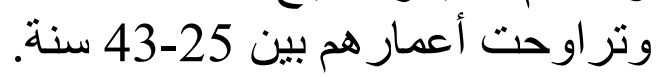

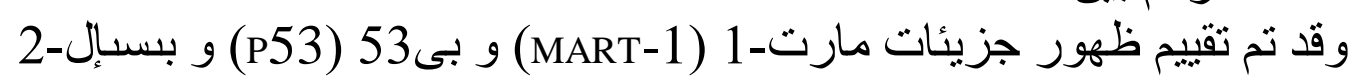

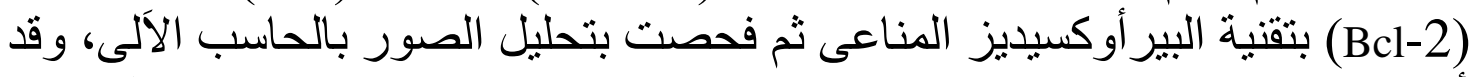

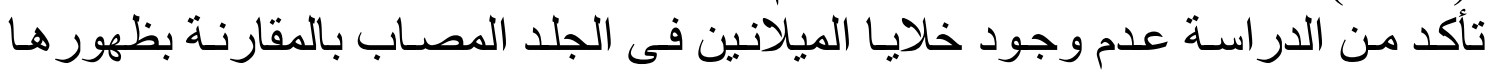

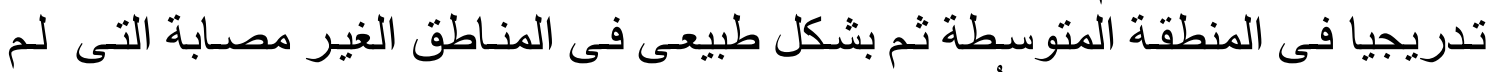

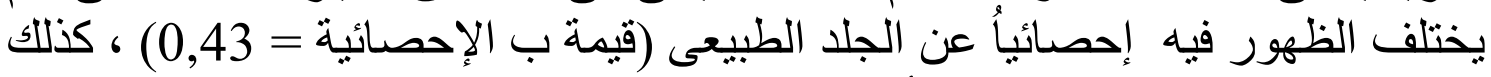

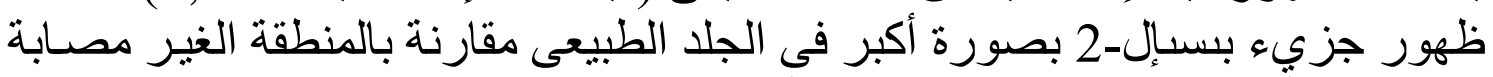

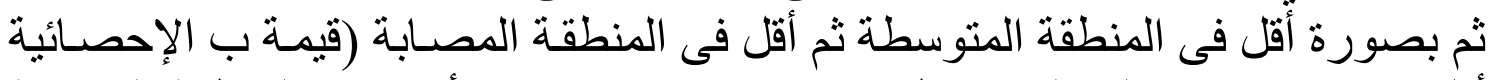

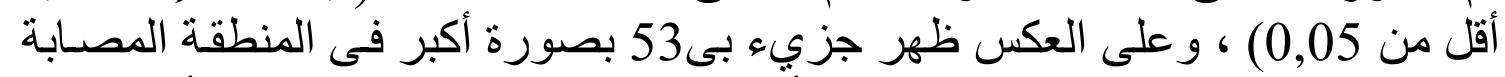

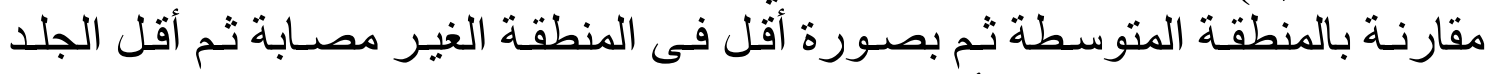

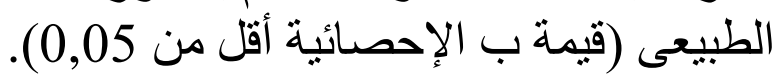

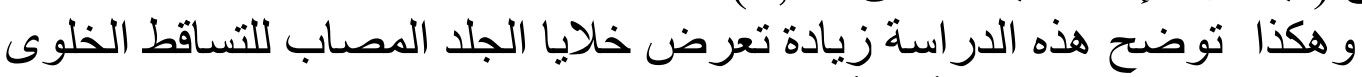

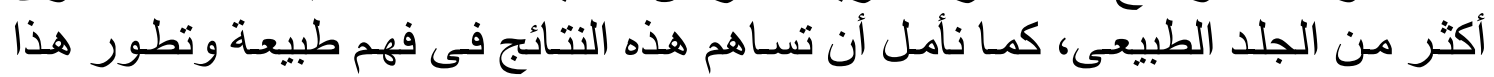
المرض و إيجاد أساليب علاجية أفضل في المئل المستقبل القريب. 